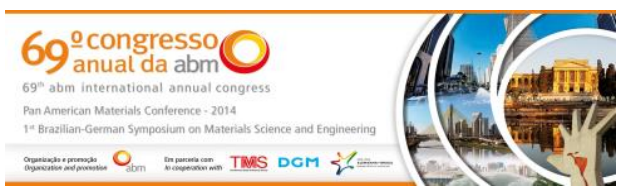

Tema: Gestão Sistêmica

\title{
A IMPORTÂNCIA DA SEGURANÇA DO TRABALHO PARA MELHORIA DA PREVENÇÃO DE ACIDENTES NA LINHA DE MONTAGEM DO SETOR AUTOMOTIVO*
}

\author{
Alexandre Rodizio Bento ${ }^{1}$ \\ Almir Rogerio Romero ${ }^{2}$ \\ Lucas Freitas ${ }^{3}$
}

\begin{abstract}
Resumo
A segurança no ambiente de trabalho é primordial para prevenir acidentes na linha de montagem do setor automotivo. Este setor busca cada vez mais a modernização tecnológica aplicada nas máquinas e a qualificação profissional para melhorar e aumentar a manufatura das peças e componentes. Para atender a demanda intensa do mercado é importante garantir o rendimento produtivo constante dos trabalhadores no chão de fábrica. Devido a esta necessidade, é fundamental exigir atenção dos trabalhadores para prevenir condições inadequadas ou acidentes. A prevenção contribui de forma positiva a empresa, com maior produtividade e ao trabalhador com melhor qualidade de vida. Neste contexto, este trabalho apresenta um estudo que aplica metodos de segurança do trabalho como forma de prevenir acidentes no setor automotivo. Os métodos aplicados na linha de montagem são detalhados e analisados para prevenir possíveis riscos de acidentes. Esta aplicação permite atingir os seguintes resultados tais como: minimizar as perdas de horas homem, maximizar a produtividade do trabalhador, reduzir custos com acidentes, melhorar a imagem da empresa perante a sociedade e a satisfação dos clientes.
\end{abstract}

Palavras-chave: Segurança do trabalho; Prevenção de acidentes; Setor automotivo.

\section{THE IMPORTANCE OF OCCUPATIONAL SAFETY IMPROVEMENT FOR THE PREVENTION OF ACCIDENTS IN THE ASSEMBLY LINE OF AUTOMOTIVE}

\begin{abstract}
A safe working environment is paramount to prevent accidents in the assembly line in the automotive sector. This sector is seeking increasingly technological modernization applied in machinery and professional qualification to enhance and increase the manufacturing of parts and components. To meet strong demand in the market it is important to ensure ongoing productive income of workers on the factory floor. Because of this need, it is essential to call the attention of the workers to prevent improper conditions or accidents. Prevention makes a positive contribution to the company, with a greater productivity and to the worker with better quality of life. In this context, this paper presents a study that applies security job methods in order to prevent accidents in the automotive sector. The methods applied on the assembly line are detailed and analyzed to prevent possible accidents risks. This application allows you to achieve the following outcomes such as: minimize loss of man hours, maximize worker productivity, reduce accident costs, improve company image in society and customer satisfaction.
\end{abstract}

Keywords: Safety, Accident prevention, Automotive sector.

1 Processamento de Dados, Mestre em Desenvolvimento de Tecnologia, Faculdades Santa Cruz, Curitiba, PR, Brasil.

2 Graduando em Engenharia de Produção, Faculdade FACEAR, Curitiba, PR, Brasil.

3 Graduando em Engenharia de Produção, Faculdade FACEAR, Curitiba, PR, Brasil.

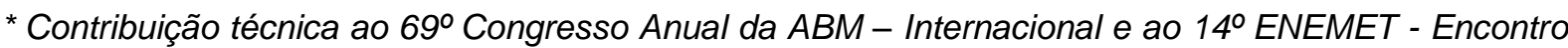
Nacional de Estudantes de Engenharia Metalúrgica, de Materiais e de Minas, 21 a 25 de julho de 2014, São Paulo, SP, Brasil.
} 


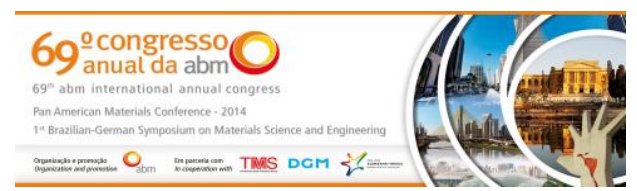

\section{INTRODUÇÃO}

A segurança do trabalho é essencial para prevenir acidentes no setor automotivo. As linhas de montagem deste setor têm alta complexidade e interação humana com várias máquinas automatizadas. Com isso, a busca por modernização tecnológica no ambiente produtivo é constante para aumentar a produtividade e reduzir custo e tempo. Esta busca aliada a qualificação profissional visa prevenir acidentes e melhorar os processos produtivos de peças e componentes automotivos com qualidade assegurada. Os investimentos em máquinas (automação) e pessoas (treinamento) fazem a indústria gerar um ambiente seguro e ao mesmo tempo um lugar de destaque no mercado.

Para atender a intensa demanda do mercado automotivo mundial é importante que a indústria esteja em constante sintonia com as normas de segurança do trabalho. Estas normas estão associadas a prevenção de acidentes no chão de fábrica e visam garantir que doenças e acidentes não atinjam o trabalhador, além de garantir o seu bem estar [1]. O trabalhador motivado em um ambiente livre de acidentes produz mais e melhor, ainda apresenta menor ausência no trabalho.

A prevenção de acidentes tem custo baixo se comparado a ocorrência do acidente que afeta diretamente o trabalhador sua família e a empresa. Desta forma, a prevenção de condições inseguras contribui de forma expressiva e positiva na imagem da empresa perante a sociedade [2]. Permite criar boas condições de trabalho e fornece maior qualidade de vida ao trabalhador.

A comissão interna de prevenção de acidentes (CIPA) visa prevenir os riscos de acidentes e doenças decorrentes do trabalho para melhorar a saúde do trabalhador [3]. A CIPA contribui em eliminar e neutralizar os riscos existentes no ambiente de trabalho. Desta forma, a legislação brasileira exige que todas as indústrias com mais de 10 funcionários formem a CIPA. Com a finalidade de assegurar que o ambiente de trabalho seja cada vez mais seguro.

Assim, a prevenção de acidentes se torna mandatório na linha de montagem do setor automotivo para evitar possíveis danos à saúde do trabalhador e consequentemente paradas da produção. A prevenção agrega valor ao produto e satisfação ao trabalhador, sendo também um dos requisitos principais da legislação do trabalho no Brasil. Neste contexto, o objetivo deste trabalho é apresentar os métodos de boas práticas de prevenção de acidentes na linha de montagem do setor automotivo. Com aplicação de algumas ferramentas de boas práticas para demonstrar a redução de acidentes e melhora da imagem da empresa perante os clientes e a sociedade.

\subsection{Segurança no Trabalho}

As empresas de vários segmentos do mercado buscam a segurança do trabalhador como fator primordial para obter lucro. Esta segurança deve ser monitorada dia a dia, devido ao avanço da tecnologia associada aos equipamentos e as mudanças que está traz nos métodos e organização do trabalho. Neste contexto, as empresas precisam disponibilizar recursos financeiros, materiais e pessoal para prevenir acidentes. A forma mais eficaz para prevenir acidentes é a implantação de programas preventivos aliado a treinamentos constantes no ambiente de trabalho.

Para aplicar as boas práticas de segurança do trabalho à empresa deve ter o serviço de segurança e medicina do trabalho (SESMT), conforme descrito na norma regulamentadora (NR) número 4, determinada pelo Ministério do Trabalho (MT).

\footnotetext{
* Contribuição técnica ao $69^{\circ}$ Congresso Anual da ABM - Internacional e ao 14ํㅡㄹ ENEMET - Encontro Nacional de Estudantes de Engenharia Metalúrgica, de Materiais e de Minas, 21 a 25 de julho de 2014, São Paulo, SP, Brasil.
} 


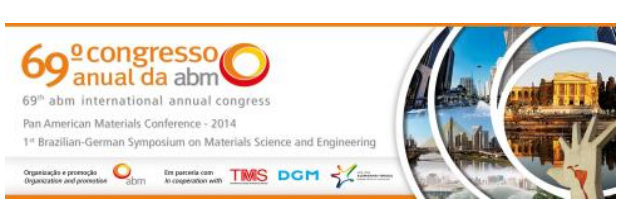

Para constituir este serviço é mandatório que os profissionais que compõe o SESMT tenham registro no $\mathrm{MT}$ e formação completa exigida perante a lei de cada profissional [4]. O número destes profissionais pode variar de acordo com o grau de risco e quantidade de trabalhadores da empresa, conforme quadro 1 do dimensionamento do SESMT descrito na NR 4.

Quadro 1. Dimensionamento do SESMT [5]

\begin{tabular}{|c|c|c|c|c|c|c|c|c|c|}
\hline $\begin{array}{c}\text { Grau } \\
\text { De } \\
\text { Risco }\end{array}$ & $\begin{array}{l}\text { Profissionais do } \\
\text { SESMT }\end{array}$ & $\begin{array}{c}50 \\
\mathrm{~A} \\
100\end{array}$ & $\begin{array}{c}101 \\
a \\
250\end{array}$ & $\begin{array}{c}251 \\
\text { a } \\
500\end{array}$ & $\begin{array}{c}501 \\
a \\
1000\end{array}$ & $\begin{array}{c}1001 \\
a \\
2000\end{array}$ & $\begin{array}{c}2001 \\
a \\
3500\end{array}$ & $\begin{array}{c}3501 \\
\text { a } \\
5000\end{array}$ & $\begin{array}{l}\text { Acima de } 5000 \text { para } \\
\text { cada grupo de } 4000 \text { ou } \\
\text { fraçāo acima de } 2000^{* *}\end{array}$ \\
\hline 2 & $\begin{array}{l}\text { Técnico Segurança Trabalho } \\
\text { Engenheiro Seg. do Trabalho } \\
\text { Aux. Enfermagem do Trabalho } \\
\text { Enfermeiro do Trabalho } \\
\text { Médico do Trabalho }\end{array}$ & & & & 1 & $\begin{array}{l}1 \\
1^{*} \\
1 \\
1^{*}\end{array}$ & $\begin{array}{l}2 \\
1 \\
1 \\
1\end{array}$ & $\begin{array}{l}5 \\
1 \\
1 \\
1 \\
1\end{array}$ & $\begin{array}{l}1 \\
1^{*} \\
1 \\
1^{*}\end{array}$ \\
\hline 3 & $\begin{array}{l}\text { Técnico Segurança Trabalho } \\
\text { Engenheiro Seg. do Trabalho } \\
\text { Aux. Enfermagem do Trabalho } \\
\text { Enfermeiro do Trabalho } \\
\text { Médico do Trabalho }\end{array}$ & & 1 & 1 & $\begin{array}{l}3 \\
1^{*} \\
1^{*}\end{array}$ & $\begin{array}{l}4 \\
1 \\
1 \\
1\end{array}$ & $\begin{array}{l}6 \\
1 \\
2 \\
1\end{array}$ & $\begin{array}{l}8 \\
2 \\
1 \\
1 \\
2\end{array}$ & $\begin{array}{l}3 \\
1 \\
1 \\
1\end{array}$ \\
\hline 4 & $\begin{array}{l}\text { Técnico Segurança Trabalho } \\
\text { Engenheiro Seg. do Trabalho } \\
\text { Aux. Enfermagem do Trabalho } \\
\text { Enfermeiro do Trabalho } \\
\text { Médico do Trabalho }\end{array}$ & 1 & $\begin{array}{l}2 \\
1^{\star} \\
1^{*}\end{array}$ & $\begin{array}{l}3 \\
1^{*}\end{array}$ & $\begin{array}{l}4 \\
1 \\
1 \\
1\end{array}$ & $\begin{array}{l}5 \\
1 \\
1 \\
1\end{array}$ & $\begin{array}{l}8 \\
2 \\
2 \\
2\end{array}$ & $\begin{array}{l}10 \\
3 \\
1 \\
1 \\
3\end{array}$ & $\begin{array}{l}3 \\
1 \\
1 \\
1\end{array}$ \\
\hline
\end{tabular}

O quadro 1 apresenta o dimensionamento do SESMT de acordo com o grau de risco e o número de empregados da empresa. $O$ grau de risco está relacionado com 0 ramo de atividade da empresa, sendo 1 a menor exposição a risco, como atividades de serviço social, comércios, escritórios e outras. Para o grau de risco 2 se enquadram empresas de educação, recreação, vestuários, fotocópias, tele atendimentos entre outras. Já para o grau de risco 3 está associada atividades hospitalar, odontólogos, usinagem, agricultura entre outras. O grau de risco 4 é o maior sendo o mesmo de alta periculosidade, onde se enquadram os segmentos de extração de carvão, minério, petróleo, cimento, fundição e outros [6].

Os profissionais de segurança do trabalho e medicina do trabalho compõem o SESMT, conforme já citado e são contratados segundo o quadro 1, ou seja, quanto maior o número de empregados e grau de risco da empresa, mais profissionais devem compor o SESMT para atender a legislação vigente. Pois, a exigência da segurança do trabalho além de ser um aspecto legal, pode estar relacionada com a melhoria na produtividade, qualidade e nas relações humanas no ambiente de trabalho.

\footnotetext{
* Contribuição técnica ao 69 Congresso Anual da ABM - Internacional e ao 14ํㅡㄹ ENEMET - Encontro Nacional de Estudantes de Engenharia Metalúrgica, de Materiais e de Minas, 21 a 25 de julho de 2014, São Paulo, SP, Brasil.
} 


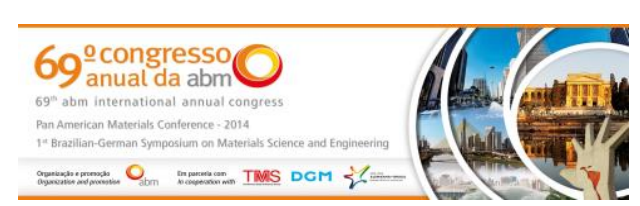

A Segurança do trabalho busca manter as perfeitas condições do ambiente de trabalho, evitar perdas humanas e materiais durante o processo produtivo da indústria. Esta preocupação visa garantir o processo de melhoria contínua e atendimento de excelência aos trabalhadores, bem como clientes internos e externos. Além disso, pode contribuir para atender os prazos de entrega de produtos e garantir a qualidade dos mesmos.

Com ambiente seguro aliado ao trabalhador qualificado e treinado com ações preventivas, pode-se evitar riscos no ambiente de trabalho [7]. A qualificação agrega cada vez conhecimento ao trabalhador no quesito de percepção de riscos de acidentes, que visa transformar o ambiente inseguro em seguro. Todos os aspectos citados contribuem em melhoria continua no trabalho e permite maior qualidade de vida ao trabalhador e seus familiares.

As empresas que tem os profissionais de segurança de trabalho podem prevenir lesões e doenças com mais eficiência. Os benefícios da segurança do trabalho no ambiente produtivo são demonstrados na figura 1.

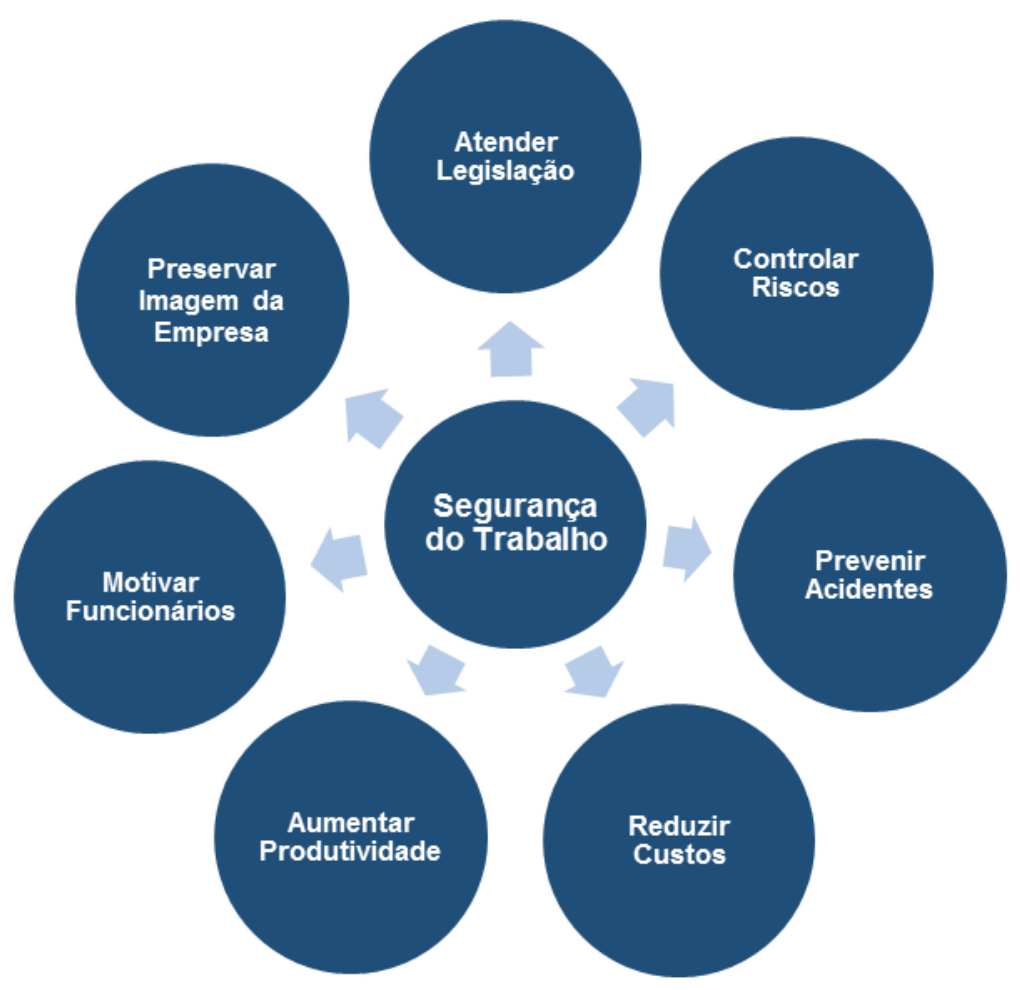

Figura 1. Benefícios da segurança do trabalho.

A figura 1 demonstra os benefícios que a empresa pode alcançar em constituir e manter o departamento de segurança do trabalho. Atender a legislação é um dos principais, pois qualquer ocorrência de acidente na empresa deve ser registrada pelo profissional de segurança do trabalho habilitado no MT. Este profissional pode contribuir para controlar os riscos no ambiente de trabalho, por meio do uso de equipamentos de proteção individual (EPI). Os EPI ajudam a prevenir acidentes e devem ser controlados pela segurança do trabalho. O controle associado ao treinamento no uso de EPI faz com que os custos com acidentes sejam minimizados e consequentemente alavancam a produtividade. Além disso, motivam os funcionários que obtêm mais qualidade de vida e ainda preserva a imagem da empresa perante a sociedade e aos concorrentes do setor.

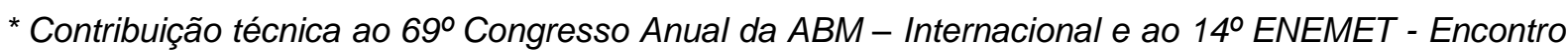
Nacional de Estudantes de Engenharia Metalúrgica, de Materiais e de Minas, 21 a 25 de julho de 2014, São Paulo, SP, Brasil. 


\subsection{Acidentes na Linha de Montagem}

Apesar da tecnologia moderna que está inserida no setor automotivo ainda são constatados vários acidentes. A tecnologia contribui para minimizar os acidentes na linha de montagem, pois muitas tarefas que exigem interferência humana foram substituídas por processos automatizados. Desta forma, investir em equipamentos robotizados na produção é fator de redução de acidentes, além disso, torna o processo mais eficiente sem falhas, devido automação empregada. Outro item positivo está relacionado à produtividade que aumenta de forma substancial, com menor custo.

Algumas linhas de montagens automotivas, ainda precisam de interferência humana para manusear peças e componentes. Estas peças normalmente têm arestas cortantes que podem ocasionar acidentes. Os acidentes na grande maioria são leves, mas já causam grandes perdas para a empresa e ao trabalhador. Além de a produção ser afetada, o cliente pode ter prejuízos que serão repassados a quem os gerou. A indústria deve atender os contratos dos clientes nas datas de entrega e quantidade de peças a serem produzidas diariamente. Já ao trabalhador o acidente traz preocupação com a saúde e aos familiares que ficam apreensivos, dependendo da gravidade do acidente e do tempo de recuperação.

Os acidentes que ocorrem na linha de montagem são investigados pela segurança do trabalho em conjunto com o responsável do setor que constatam a origem do acidente se foi um caso de negligência, imperícia ou ato inseguro [8]. A investigação descreve um relatório com todos os dados dos acidentes que são registrados e servem como base descobrir o que ocasionou o mesmo, também visa resolver pontos de falhas para que a ocorrência não volte acontecer.

Segundo Bird Jr. e Germain [9] o acidente é uma ocorrência não desejada que ocasiona dano a pessoa ou empresa. Os mesmos autores ressaltam se o acidente resultar apenas em dano a propriedade ou perda no processo e não ocasionar lesão, ainda é um acidente. Desta forma, o acidente não está relacionado apenas ao ser humano, mas também a ocorrência na propriedade como incêndio, quebra de ferramentais e máquinas entre outros.

Para Benito, Araújo e Souza [10] o acidente que ocorre no exercício do trabalho pode gerar lesão corporal, perturbação, doença, morte, redução da capacidade de trabalhar temporária ou permanente. Mesmo uma das causas citadas não seja a única, mas tenha contribuído para o resultado de prejuízo físico do trabalhador pode ser chamado de acidente.

Para minimizar ou evitar os acidentes nos ambientes de trabalho o uso das boas práticas de prevenção surge como fator primordial nas indústrias automotivas. Pois aos prejuízos causados no trabalhador e na indústria são maiores que prevenir e conscientizar dos possíveis riscos. As origens e causas de ocorrências indesejáveis no ambiente de trabalho podem ser divididas em quatro grandes elementos sendo a pessoa (elemento humano), o equipamento (máquinas e ferramentas usadas no trabalho), os materiais (produtos e substâncias que são manipuladas) e o meio ambiente (representado pelos riscos químicos e biológicos). Todos os elementos descritos devem interagir entre si de forma adequada para não ocasionar perdas [11].

Para inserir as boas práticas para prevenir acidentes na linha de produção a participação e comprometimento da alta direção é fator primordial. Pois a cultura de segurança deve ser de responsabilidade de todos os trabalhadores do chão de fábrica até o presidente da indústria [12]. Para ter um ambiente de trabalho seguro

\footnotetext{
* Contribuição técnica ao $69^{\circ}$ Congresso Anual da ABM - Internacional e ao 14ํㅡㄹ ENEMET - Encontro Nacional de Estudantes de Engenharia Metalúrgica, de Materiais e de Minas, 21 a 25 de julho de 2014, São Paulo, SP, Brasil.
} 


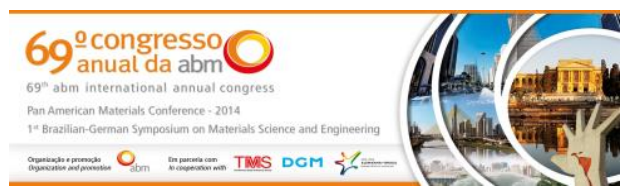

A figura 2 no item (a) demonstra o fluxo do AS o qual é emitido quando um funcionário identifica uma ocorrência na linha de montagem, esta pode ser um risco de acidente ou um ato inseguro gerado por falha humana ou máquina. Para preencher o AS o funcionário relata todos os problemas/falhas identificadas e coleta assinatura do responsável do setor da ocorrência. Com o AS preenchido e assinado deve ser entregue no setor de segurança do trabalho que procura investigar o relatado no AS e descreve um relatório com uma ação para corrigir o problema e evitar um possível acidente.

Já o item (b) da figura 2 apresenta o fluxo do DS que é realizado uma vez por semana com duração de até 15 minutos na própria linha de montagem. A implantação do DS deve ter o apoio e participação da gerência de fábrica em todas as reuniões iniciais em conjunto com a segurança do trabalho. A participação demonstra compromisso aos funcionários pela gerência. O DS é realizado pelo supervisor da linha que alguns dias antes da reunião define o tema em conjunto com a segurança do trabalho, com base nas ocorrências evidenciadas no setor. Após apresentar o tema e orientar todos os funcionários do problema, são solicitadas possíveis sugestões para solucionar a ocorrência relatada. Todo DS é evidenciado em ata com a ocorrência e as possíveis sugestões e todos os funcionários da linha devem assinar o mesmo. Após o documento assinado deve ser entregue na segurança do trabalho que registra e analisa os problemas/sugestões, e faz um plano de prevenção das ocorrências para trazer mais segurança ao trabalhador na linha de montagem.

\section{RESULTADOS E DISCUSSÃO}

Para demonstrar os resultados da implantação das boas práticas de prevenção de acidentes a partir de 2012 foi utilizado o AS e DS nas linhas de montagem, conforme tabela 1 com os números de acidentes registrados em cada ano.

Tabela 1. Acidentes registrados nas linhas de montagem por ano

\begin{tabular}{lccccc}
\hline Linhas de Montagem & Qtde 2011 & Qtde 2012 & $\begin{array}{c}\text { \% Redução } \\
2012\end{array}$ & Qtde 2013 & $\begin{array}{c}\% \text { Redução } \\
2013\end{array}$ \\
\hline Bloco de Motor & 51 & 21 & $59 \%$ & 11 & $48 \%$ \\
\hline Cabeçote & 59 & 39 & $34 \%$ & 14 & $64 \%$ \\
\hline Biela & 46 & 20 & $57 \%$ & 7 & $65 \%$ \\
\hline Carcaça Diferencial & 25 & 5 & $80 \%$ & 4 & $20 \%$ \\
\hline \multicolumn{1}{c}{ Total Geral } & 181 & 85 & $53 \%$ & 36 & $58 \%$ \\
\hline
\end{tabular}

A tabela 1 apresenta os registros dos acidentes a partir de 2011 onde é possível observar a redução expressiva no número de acidentes em 2012 e 2013 com a implantação e utilização das ferramentas de prevenção de acidentes AS e DS. Em 2012 a redução de acidentes das linhas de bloco de motor atingiu o índice de 59\%, de cabeçote $34 \%$, biela $57 \%$ e carcaça diferencial $80 \%$, todas em relação a 2011 . Observa-se que todas as linhas de montagem tiveram redução expressiva no número de acidentes devido aplicação do AS e DS.

Já em 2013 os percentuais de acidentes de todas as linhas continuam em declínio acentuado em relação aos números atingidos em 2012. As linhas com índices mais expressivos de redução foram: o cabeçote $(64 \%)$ e biela (65\%). Os altos percentuais

* Contribuição técnica ao 69 Congresso Anual da ABM - Internacional e ao 14ํㅡㄹ ENEMET - Encontro Nacional de Estudantes de Engenharia Metalúrgica, de Materiais e de Minas, 21 a 25 de julho de 2014, São Paulo, SP, Brasil. 
de redução estão associados ao sucesso da utilização das fermentas de boas práticas de prevenção de acidentes AS e DS. Outro fator importante é o monitoramento constante dos quase acidentes pela segurança do trabalho com plano de ação eficaz.

A figura 3 representa na forma gráfica a redução no número de acidentes por linha de montagem e ano, onde pode se observar que em 2013 totalizou 36 acidentes, ou seja, 58\% de redução em relação a 2012 e 80\% para 2011.

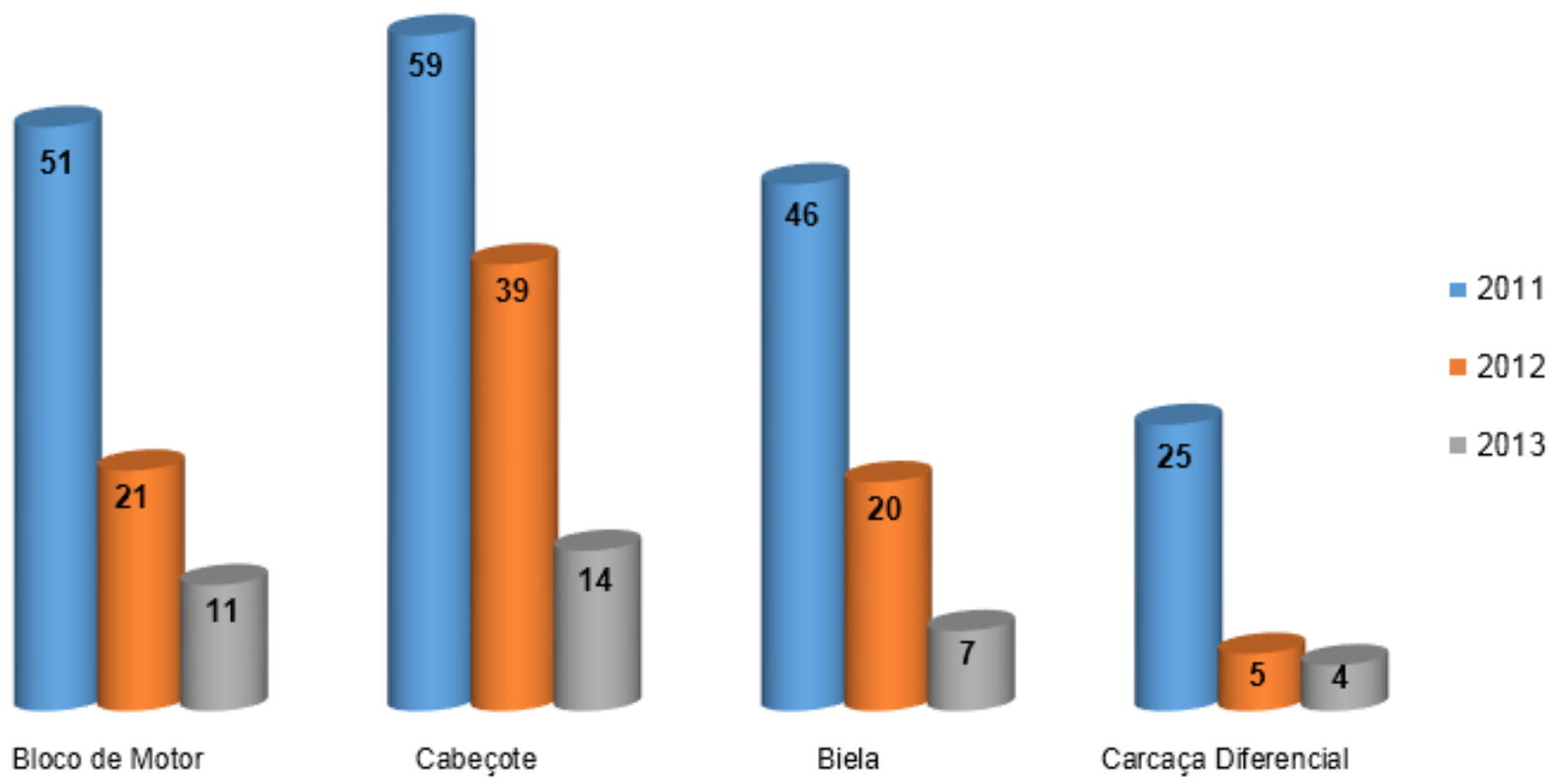

Figura 3. Número de acidentes por linhas e ano.

Ao se observar a figura 3 e o quadro 1 apresentados, pode-se verificar que a quantidade de acidentes por ano teve redução acentuada. Se comparar esta quantidade versus ao tempo de uma hora que o funcionário fica em média no atendimento médico, este número de horas é expressivo. Em 2011 o total de acidentes foi 181 , ou seja, 181 horas paradas a um custo de $R \$ 9,00$ a hora por trabalhador ou $\mathrm{R} \$ 1.629,00$ reais somente com a saída do funcionário da linha de montagem para atendimento médico dentro da indústria. Além disso, a quantidade de peças produzidas também diminui o que pode gerar horas extras futuras para atender à necessidade do cliente.

Assim os custos relacionados aos acidentes de trabalho são expressivos e difíceis de serem calculados, pois se deve somar o valor do tempo que o funcionário permanece fora do local de trabalho, mais a redução na quantidade produzida da linha de produção (máquina ociosa) e outros gastos com atendimentos. O tempo em horas dos acidentes sem afastamento diminuíram na mesma velocidade que a quantidade dos mesmos durante os anos analisados. Em 2012 o custo referente a 85 horas paradas foi de $R \$ 765,00$ reais, já em $2013 R \$ 324,00$ reais ou seja 36 horas.

O cenário analisado observa-se que os acidentes de trabalho e as horas de afastamento do trabalhador tem diminuído na mesma proporção de forma rápida. Devido a implementação dos métodos de boas práticas de prevenção de riscos de acidentes. Esta implementação permite maximizar a produtividade do trabalhador, maior qualidade de vida ao trabalhador, minimizar custos com acidentes, gerar um

\footnotetext{
* Contribuição técnica ao 69ำ Congresso Anual da ABM - Internacional e ao 14ํㅡㄹ ENEMET - Encontro Nacional de Estudantes de Engenharia Metalúrgica, de Materiais e de Minas, 21 a 25 de julho de 2014, São Paulo, SP, Brasil.
} 


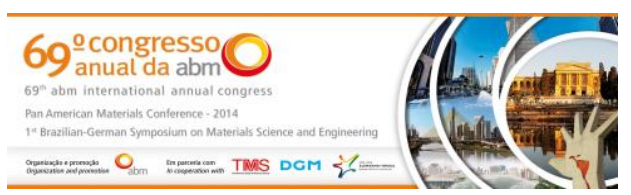

ambiente livre de acidentes, melhorar a imagem da indústria e atender as demandas dos clientes no prazo com qualidade.

\section{CONCLUSÃO}

A implantação de ferramentas para prevenir acidentes de trabalho é uma exigência na indústria automotiva. Devido a necessidade de atender a legislação e também cumprir os contratos dos clientes. Os acidentes trazem danos à saúde do trabalhador e à indústria, com possíveis reduções na quantidade de peças produzidas, aumentando o custo final da peça. Estes fatores podem trazer complicadores na parceria entre fornecedor e cliente. Neste cenário surgem as boas práticas de prevenção de acidentes que agregam mais segurança ao trabalhador no ambiente de produção.

O setor automotivo busca eliminar os acidentes de trabalho nas linhas de montagem por meio da prevenção e uso de práticas seguras. Os acidentes têm alto custo agregado para as indústrias, devido ao avanço das legislações do trabalho. Assim a prevenção dever ser primordial no ambiente fabril, tendo como aliada a segurança do trabalho que busca modernas ferramentas para contribuir de forma expressiva para reduzir os riscos de acidentes na linha de montagem.

A aplicação das boas práticas de prevenção de acidentes mostrou-se superior na redução de acidentes com índices baixos nos anos analisados em 2012 a redução de 58\% em relação a 2011 nas linhas de montagem selecionadas, Já em 2013 a redução foi de $53 \%$ em relação a 2012. Analisando o último ano com o primeiro o percentual de redução de acidentes chega aos $80 \%$. Todos estes índices trazem benefícios para a indústria, e de forma orquestrada ajudam a eliminar ou minimizar os acidentes nas linhas de montagem.

Além dos ganhos já citados, aplicar as boas práticas de prevenção de acidentes nas linhas de montagem podem permitir atingir ganhos diferencias nas peças e componentes automotivos. Com isso, garante melhorias e investimento na produção, principalmente onde os riscos de acidentes ainda são existentes. Todos os aspectos mencionados contribuem para melhora da imagem da indústria perante os clientes e a sociedade.

\section{REFERÊNCIAS}

1 Ferreira MM, Souza CES, Ribeiro CA, Galdino DB, Ricci GL. Avaliação Sobre a Prevenção de Riscos na Atividade de Trabalho em Prensas. Iberoamerican Journal of Industrial Engineering. 2012;4:48-68.

2 Marciane D, Stefano SR, Limongi-Franca AC. Acidentes de Trabalho: um velho desafio. In: SEMEAD; 2008; São Paulo, Brasil. São Paulo: FEA, USP; 2008.

3 Garcia GFB. Segurança e Medicina do Trabalho - Legislação. 3ª ed. São Paulo: Método; 2010.

4 Araujo GM. Normas Regulamentadoras Comentadas e llustradas: legislação de segurança e saúde no trabalho. $11^{\text {a }}$ ed. Rio de Janeiro: GVC; 2014.

5 Manuais de legislação. Segurança e Medicina do Trabalho. $67^{a}$ ed. São Paulo: Atlas; 2011.

6 Curia LR, Windt MCVS, Cespedes L. Segurança e Medicina do Trabalho. São Paulo: Saraiva; 2011.

7 Chaib EBD. Proposta para Implementação de Sistema de Gestão Integrada de Meio Ambiente, Saúde e Segurança do Trabalho em Empresas de Pequeno e Médio Porte:

\footnotetext{
* Contribuição técnica ao 69ำ Congresso Anual da ABM - Internacional e ao 14ํㅡㄹ ENEMET - Encontro Nacional de Estudantes de Engenharia Metalúrgica, de Materiais e de Minas, 21 a 25 de julho de 2014, São Paulo, SP, Brasil.
} 
um estudo de caso da indústria metal-mecânica [dissertação de mestrado]. Rio de Janeiro: Universidade Federal do Rio de Janeiro; 2005.

8 Saurin TA, Ferreira CF. Diretrizes para Avaliação dos Impactos da Produção Enxuta Sobre as Condições de Trabalho. Produção (São Paulo. Impresso). 2008;18:127-141.

9 Bird Jr. FE, Germain G. Liderança Prática em Controle de Perdas. Georgia: International Loss Control Institute; 1990.

10 Benito J, Araujo GM, Souza CR. Normas Regulamentadoras Comentadas. $2^{\underline{a}}$ ed. Rio de Janeiro: GVC; 2000.

11 Lick VLC. Melhoria das Condições de Trabalho Através da Ação Ergonômica Participativa e da lógica do PDCA no Setor Automotivo [dissertação de mestrado]. Porto Alegre: Programa de Pós-Graduação da Escola de Engenharia, Universidade Federal do Rio Grande do Sul; 2003.

12 Choudhry RM, Fang D, Mohamed S. The nature of safety culture: A survey of the stateof-the-art. Safety Science. 2007;45:993-1012.

* Contribuição técnica ao 69 Congresso Anual da ABM - Internacional e ao 14ํㅡㄹ ENEMET - Encontro Nacional de Estudantes de Engenharia Metalúrgica, de Materiais e de Minas, 21 a 25 de julho de 2014, São Paulo, SP, Brasil. 